\title{
Optical Fiber Imaging for High Speed Plasma Motion Diagnostics: applied to Low Voltage Circuit Breakers
}

\author{
J.W.McBride*, A. Balestrero++, L. Ghezzi+++, G. Tribulato++, K.J. Cross+
}

*School of Engineering Sciences, University of Southampton, Highfield, Southampton SO17 1BJ, United Kingdom, J.W.McBride@soton.ac.uk

+ Taicaan Ltd, 2 Venture Road, Southampton Science Park, Southampton, Hampshire, SO16 7NP, United Kingdom, info@taicaan.com

++ ABB S.p.A., ABB SACE DIVISION, Via Baioni, 35 IT-24123, Bergamo

+++ ABB S.p.A., ABB SACE DIVISION, Viale dell’Industria, 18 IT-20010, Vittuone (MI)

\begin{abstract}
An integrated portable measurement system is described for the study of high speed and high temperature unsteady plasma flows such as those found in the vicinity of high current switching arcs. An array of optical fibers allows the formation of low spatial resolution images, with a maximum capture rate of 1 million images per second (1MHz), with 8 bit intensity resolution. Novel software techniques are reported to allow imaging of the arc; and to measure arc trajectories. Results are presented on high current $(2 \mathrm{kA})$ discharge events in a model test fixture and on the application to a commercial low voltage circuit breaker.
\end{abstract}

\section{Introduction}

There are many applications in aerospace, combustion and electrical systems where the medium of interest is a gas at high temperature in an unsteady flow regime. Using high speed imaging and image-processing techniques, spatially and temporally resolved parameters including object location, size, area, number, velocity and direction of movement can be determined. A system is described for the study of high speed and high temperature unsteady plasma flows such as those found in the vicinity of high current switching arcs. Recent studies into the phenomena have included a technique for broadband optical absorption spectroscopy for the investigation of transient media in low-voltage circuit breakers, [1]. In [2] a combination of systems were used to study arc motion; including a conventional high-speed camera providing 6000 images/s, a CCD camera with 105ns exposure time but a limited number of 15-20 images, and magnetic diagnostic tool. The experimental measurements and theoretical calculations were used to identify and quantify the influence of the arc chamber insulation has on post-current zero recovery voltage. In [3], gas analysis, pressure measurements, and post currentzero breakdown voltages were used to rank arc chamber wall material according to breakdown voltage. A previous optical 
fiber based imaging system was developed in the early 1990's and was used in studies of arc motion, [4-9]. The system was limited to 500MHz sampling over a 8ms duration with 6 bit light intensity resolution. Critically there was no control of the system sensitivity and the methods developed to characterize arc motion have since been shown to be subject to displacement errors.

In order to optimize the design and development of circuit breaker technology, it is essential to gain an understanding of the events that occur as the arc moves from a contact region, where the arc is initiated by opening contacts; through an arc chamber to the arc stack or splitter plates. Fig.1 shows an example system with the opening contact region at the top of the chamber and the splitter plates at the bottom of the chamber. In the figure the cathode is the fixed contact region on the right and the anode is the moving contact region of the left. The arc motion through chamber is the result of electro-magnetic forces driving the plasma column into the plates. The physics of the transfer process through the arc chamber is a complex multi-physics problem. When the arc reaches the splitter plates the voltage across the arc increases as a consequence of the multiple anode and cathode fall voltages, [4]; this increase in voltage acts to limit the current and deionizes the plasma.

\section{The Arc Imaging System (AIS)}

The optical fiber used for the system is standard plastic fiber with $1 \mathrm{~mm}$ diameter and $0.6 \mathrm{~mm}$ sheath $(2.2 \mathrm{~mm}$ overall diameter); it has $-0.15 \mathrm{~dB} / \mathrm{m}$ attenuation at $525 \mathrm{~nm}$ wavelength, and the transmission attenuation is lowest in the 400 to 700nm, visible light range. In the system presented the fiber length is $5 \mathrm{~m}$, allowing electrical isolation between the arcing event and the data acquisition. Fig.1. shows an example test chamber, used to investigate the relationship between contact opening velocity and the cathode and anode arc root dynamics as the arc transverses the arc chamber, [6-9]. The arc chamber is mounted in the horizontal plane, and is designed to simplify the geometry of a typical chamber. The anode and cathode conductors are shown with a steel backing plate to enhance the electro-magnetic influence. The circles in Fig. 1 show the positions of the optical fibers in the array; referred to as the optical head. The optical head is mounted behind a quartz glass section which seals the arc chamber and connected to the data logging system using two bespoke connectors. To calibrate the system, each optical fiber position is tested with a reference LED light source, and a calibration factor stored. In the data logging system, each optical fiber is coupled to a photo-diode, and programmable amplifier (gain x1 to $\mathrm{x} 32$ ) allowing for full system sensitivity control. The system is designed to sample at $1 \mathrm{MHz}$ with 8 bit resolution, providing 256 intensity levels. The electronics are mounted with 16 addressable channels per card, and designed to accommodate up to 64 cards. In the results presented a 68 channel (see Fig.1) and a 50 channel optical head are used. In earlier systems the 
memory was typically limited to $4 \mathrm{~K}$, allowing $8 \mathrm{~ms}$ of data at $500 \mathrm{kHz}$, [4]. In the current system the memory allocation is $512 \mathrm{~K}$, allowing 0.5 seconds of data at $1 \mathrm{MHz}$.

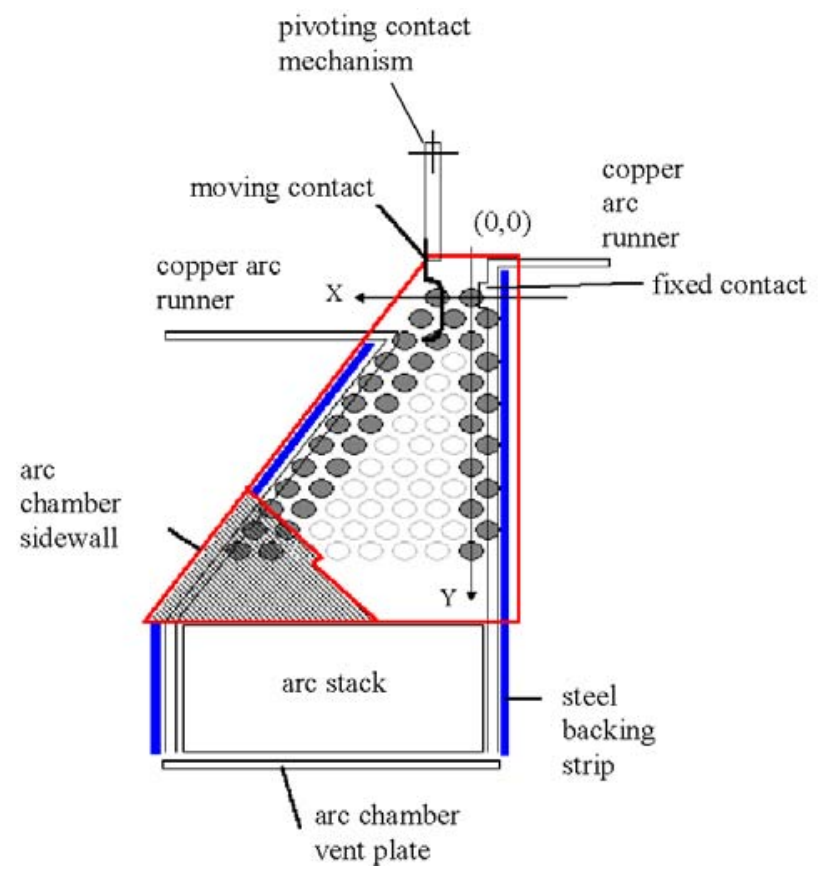

Fig.1 Schematic of the arc chamber with 68 fiber positions. The shaded fiber positions correspond to the fiber selected for arc root trajectory plotting. The cathode is connected to the fixed contact runner.

\subsection{The Light Intensity data}

Fig 2 shows image of the arc chamber in Fig.1, with the address of each fiber position; it shows the contact region on the right and the splitter plates on the left. The fiber positions along the cathode arc runner, also shaded in Fig.1, are labeled in sequence from the moving contact region on the right: B, E, H, L, P, U, Z, AF, AL, AS, AZ, BH and BR. Fig.3 shows an example of the calibrated light intensity data from fiber positions E, L, and $\mathrm{U}$ for a single test in the arc chamber discussed in the results section. The light intensity levels from fiber $\mathrm{E}$ in the contact region increases to 100 at $2670 \mu$ s; for fiber $\mathrm{L}$ and U the corresponding time are respectively $2850 \mu$ s and $2870 \mu$ s. The peak intensity for fiber $\mathrm{E}$ is 150 while the corresponding values for $\mathrm{L}$ and $\mathrm{U}$ are respectively 270 and 320.

\subsection{Arc Imaging}


There are two modes of arc imaging; mode 1 uses dynamic thresholds; while mode 2 uses fixed thresholds. With dynamic thresholds the maximum value is the maximum light intensity across all the fibers at a given frame number or time. For the three fibers in Fig 3, at $2800 \mu$ s the maximum intensity is 120 in fiber E. With fixed thresholds the maximum value is the maximum light intensity across all the fiber for all frames; for the data shown in Fig.3 the maximum value is 320 . For the former the images are created with a different maximum intensity for each frame, this is best suited for viewing the arc when the light intensities are low; for example at the start of the arc. The latter is best suited for an overview of the arc process when the arc is in the chamber, as the image intensity scale will be fixed, allowing a systematic comparison of arcing events. In addition to the mode of imaging there are a number of methods for creating the images.

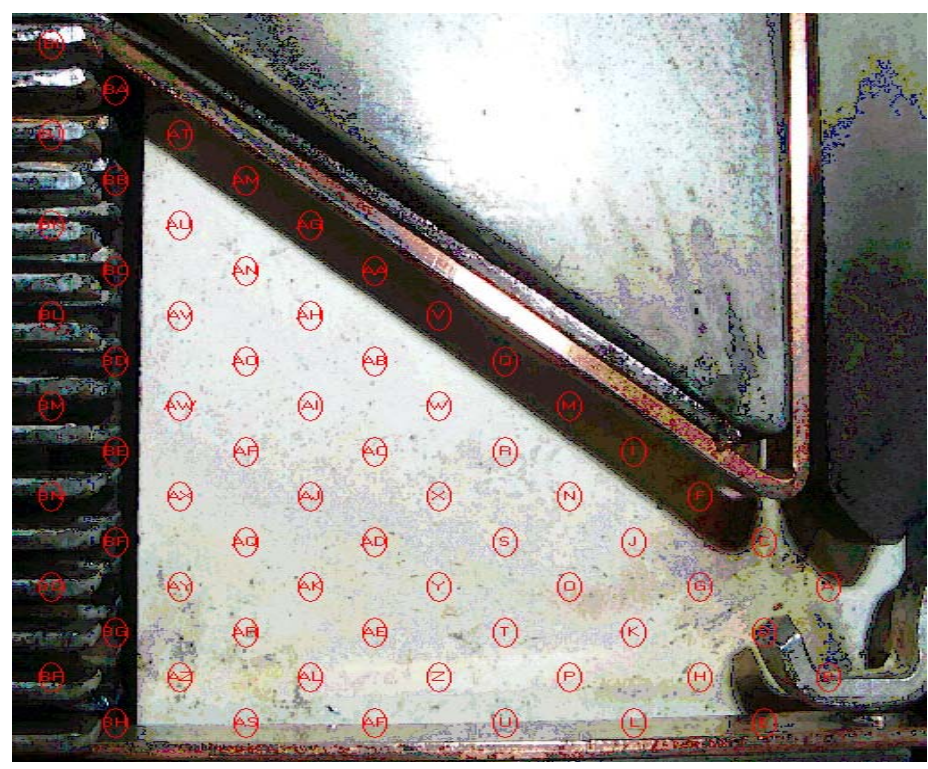

Fig 2. The addressing of fiber positions in the optical array. 


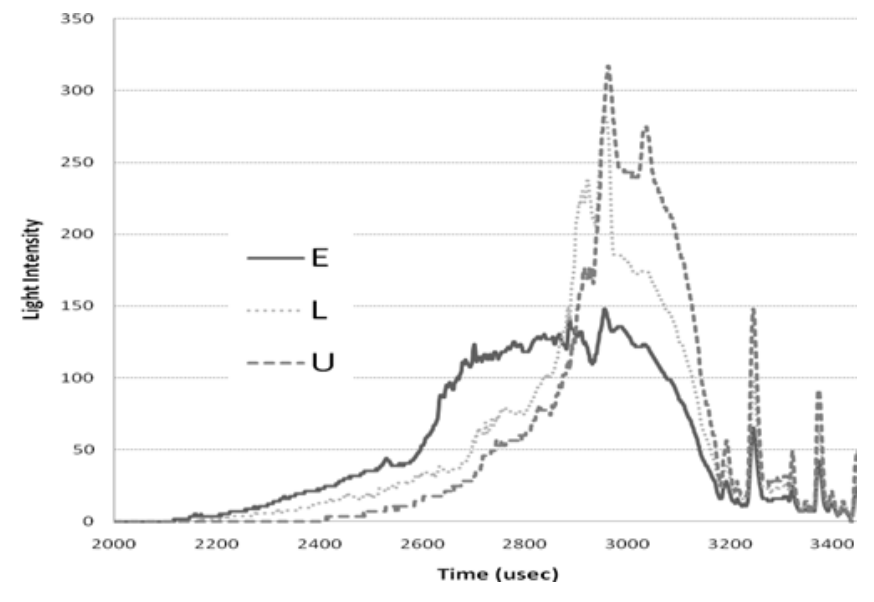

Fig 3. Light intensity for selected fiber (experiment 1).

Method 1. Group Contours. Contours are grouped for the whole array which then creates an image of the arc. The method has been described previously in [4], using 6bit light intensity data. The method is ideally suited to the low intensity resolution data, however with increased bit resolution a number of new methods of imaging have been developed.

Method 2. Point Contours. Each fiber position is illuminated with color coded concentric circles, where the color of each embedded circle is related to a percentage of the maximum light intensity level, using either mode 1 or 2 to define the maximum level. The key advantage over method 1 is that, with the increased bit resolution of the light intensity, multiple conducting paths are detectable.

Method 3. Simulated images. This allows the creation of realistic images of the arc discharge process at sample rates not possible with conventional video or photographic methods. The fiber spots are interpreted as the nodes of a planar triangulation, which is automatically produced by means of the classical Delaunay algorithm [11]. The light intensity in the points of each triangle of the triangulation is estimated by linear interpolation and is then mapped to the color scale accordingly. Particularly, if $V_{j}, j=1,2,3$, are the vertices of a triangle $T$ containing a point $P$, and if $I_{j}, j=1,2,3$, are the light intensity values in the three vertices, respectively, then the light intensity value in point $P$ is

$$
I=\frac{\sum_{j=1}^{3} I_{j} A_{j}}{A},
$$


where $A_{j}$ is the area of the sub-triangle with vertices $P$ and the two vertices of $T$ other than $V_{j}$, and $A=\sum_{j} A_{j}$ is the total area of $T$. It is immediately seen that, when $P$ lies onto some edge of $T$, then the classical $1 \mathrm{D}$ linear interpolation scheme over a line segment is attained, and if $P=V_{j}$, for some $j$, then $I=I_{j}$. According to this scheme, all of the points belonging to the convex hull of the collection of fiber spots are given a suitable color. The linear interpolation assumption is obviously arbitrary and care must be paid when examining and interpreting the visual result, since any interpolation scheme is unsuitable whenever some obstacle, such as the solid portion of a splitter plate, is interposed between two fiber spots.

\subsection{Arc Root Plotting}

An established method of arc root plotting allows for the definition of parameters, for example the time taken for the arc root to transfer from the moving contact to the fixed runner; such that physical variables, for example the materials used in the chamber can be investigated and compared, [4-9]. The method is based on an intensity based position averaging technique over selected fiber positions near the surface of the runners (shaded fibers in Fig 1). The method normalizes the sum of the products of the fiber intensity, and fiber position; as shown in (2), where the resultant $\mathrm{x}$, and y positions are in the axis indicated in Fig.1, while the $X_{i}, Y_{i}$ values are the positions of each optical fiber in the selected array.

$$
x=\frac{\sum_{j \in J_{\text {root }}} I_{j} X_{j}}{\sum_{j \in J_{\text {root }}} I_{j}}, \quad y=\frac{\sum_{j \in J_{\text {root }}} I_{j} Y_{j}}{\sum_{j \in J_{\text {root }}} I_{j}},
$$

where; $J_{\text {root }}$ is a suitable index set, referencing those fibers located along a given arc runner.

A limitation of the application of (2) not previously identified is in the influence of low light levels when $\mathrm{X}$ or $\mathrm{Y}$ are large. This results in an error in the position of the arc root. This was not important in the previous studies as the parameters were used in comparative studies. However to understand the link between the absolute position of the arc root and the physical process of the arc root on the runner surface requires a modification to the method. To overcome this error, and to determine the positional resolution of the arc root, the additional intensity bit resolution (0-255) allows filter values to be used such that the position of the arc root is more clearly defined. In this new approach low level intensities are filtered such that the position of the cathode arc root can be accurately determined, and then correlated to physical processes. For the cathode root in Fig. 1 the first fiber position $\mathrm{B}$ is allocated $\mathrm{Y}=1 \mathrm{~mm}$, and the $\mathrm{AZ}$ fiber $\mathrm{Y}=40 \mathrm{~mm}$. 


\section{Experimental methods}

\section{Experiment 1. Flexible Test Apparatus.}

The test system used has been described in [4]. In brief, the flexible test apparatus shown in Fig.1 is coupled with a capacitive discharge system, such that current starts to increase 2ms prior to the opening of the contacts; a peak short circuit current of approximately $2 \mathrm{kA}$ occurs with a half cycle period of 10ms. New arc runners and contact materials were selected for each test. In all cases the cathode was consistent with Fig.1, the vent was $15 \%$ open, and the moving contact was connected to a high speed solenoid opening device, ( $>6 \mathrm{~m} / \mathrm{s})$. After a single test operation the arc runners are removed from the apparatus. To correlate the arc images with the surface interaction along the arc runner surface, an optical surface profiler is used. The optical profiler is a XYRIS4000CL; it uses a con-focal laser probe to create detailed 3D images of the surface of the arc runners, [12].

\section{Experiment 2. Application to a Commercial Low Voltage Circuit Breaker.}

The optical head with an array of 51 fibers is mounted on one side of the circuit breaker under test, as shown in Fig. 4 . The position and diameter of the holes in the sidewall of the breaker are critical. The holes cannot be drilled in locations where there is a splitter plate or other components which would interfere with the light transmission path. The distance of the end of each optical fiber from the arc chamber and the diameter of the hole in the optical head is important. If the distance is increased the field of view of each fiber will reduce, [4]. This is often a desirable result and can be counterbalanced by the adjustable sensitivity of the imaging system. 


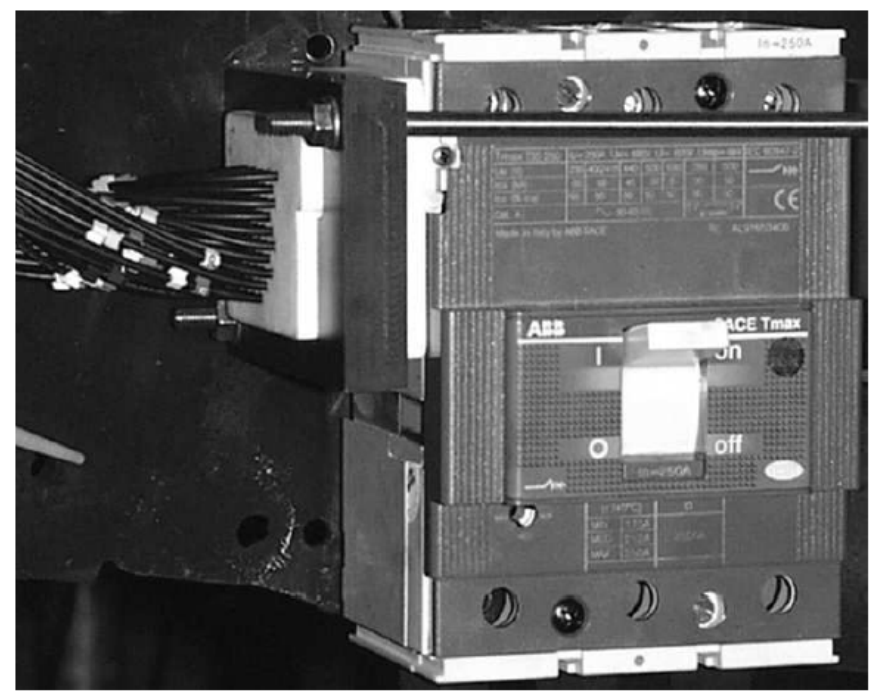

Fig.4 Low voltage circuit breaker equipped with optical head.

\section{Results}

\subsection{Experiment 1.}

The arc voltage and current are shown in Fig.5. The time base for the data is referenced from the start of the short circuit current pulse. The cathode root position is scaled and shown on the same axis as the arc voltage. The maximum light intensity is also shown. The opening of the contacts corresponds to the step in arc voltage at $1.95 \mathrm{~ms}$, when the arc current is 1.3kA. The step in the arc voltage is followed by an increase in voltage as the plasma develops and is forced through the arc chamber towards the splitter plates. The arc voltage peaks at approximately $270 \mathrm{~V}$ as it enters the splitter plates, at $3.3 \mathrm{~ms}$, leading to the reduction in the arc current. Fig.5 shows the maximum light intensity from the fibers selected to detect the cathode root motion (B to BF). The intensity rises after the opening of the contacts and reflects the corresponding increases in the arc power. For the same experiment, Fig 6a shows the arc column using imaging method 2, at 2.947ms having transferred from the moving contact to the anode arc runner, and conducting to the cathode runner region, outside the fixed contact region. The contour values selected are between $30-42 \%$ of the maximum light intensity from fiber $\mathrm{U}$, shown to be 320 in Fig.3. Fig 6b shows the arc $12 \mu$ s later, at 2.959ms, with the arc column now in the middle of the chamber. This image shows the arc column with a single root on the cathode, but with multiple conducting paths to the anode surface. 


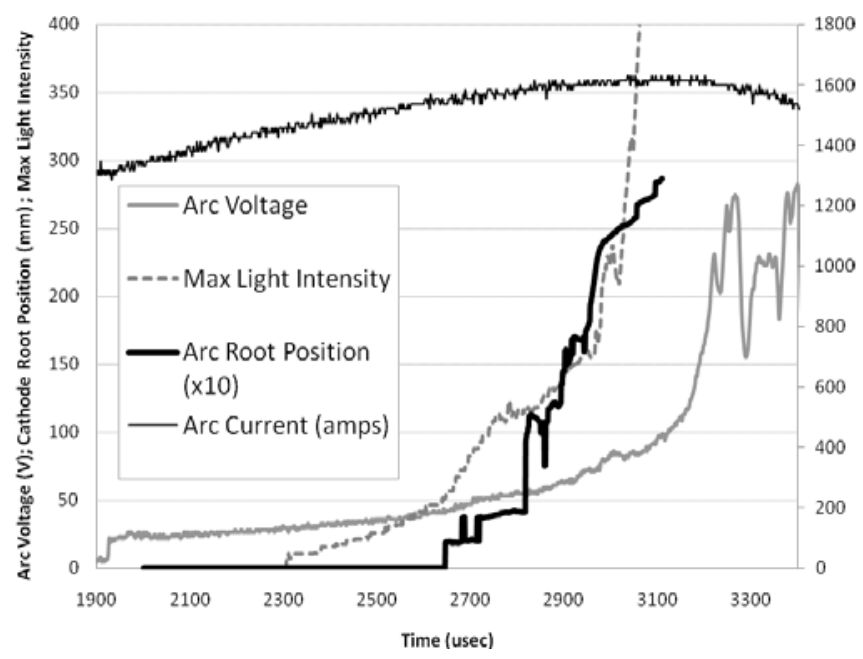

Fig.5 Left Axis: Arc Voltage(V), with Maximum Light Intensity and Cathode Arc Root Position (x10mm), using a filter value of 90; Right Axis: Arc Current (A)

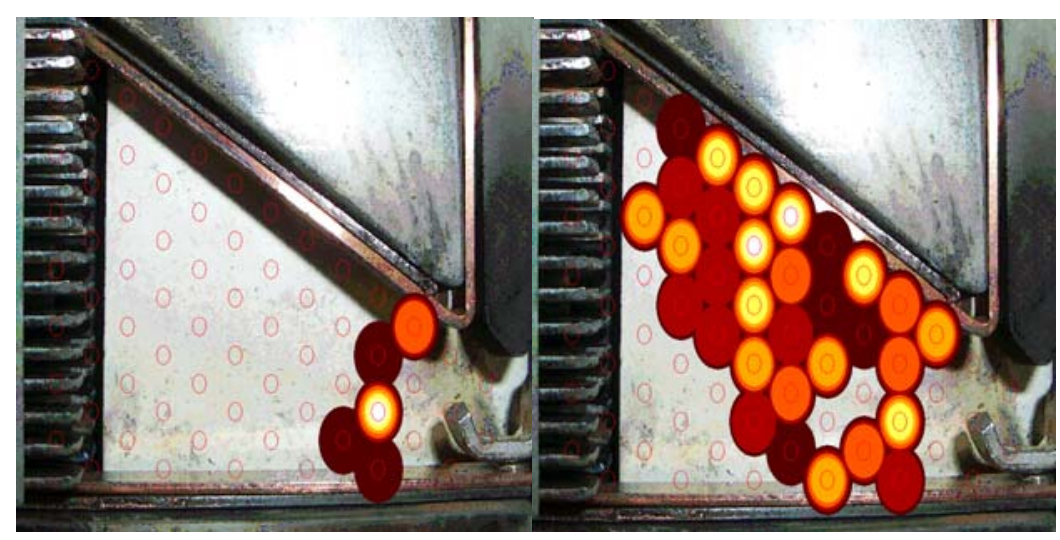

(a)

(b)

Fig. 6 Arc images using selected filters based on maximum value from fiber $U$, $U(\max )$; at a)2.947ms, and b)2.959ms, using contour values corresponding to 30,32,34,36,38,40,42\%, of $U(\max )$. 


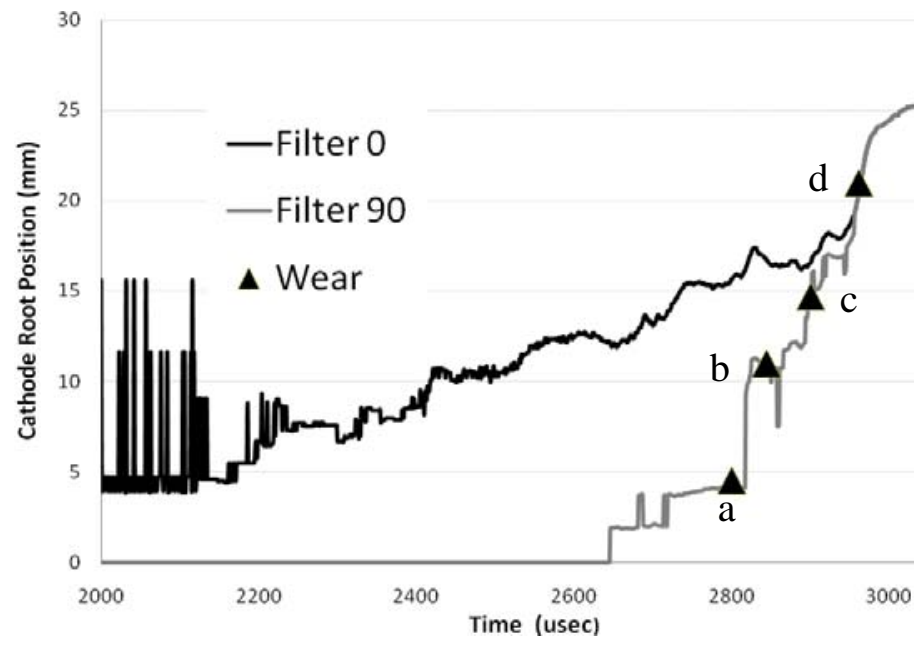

Fig.7 The influence of cathode root motion with filter values 0 .

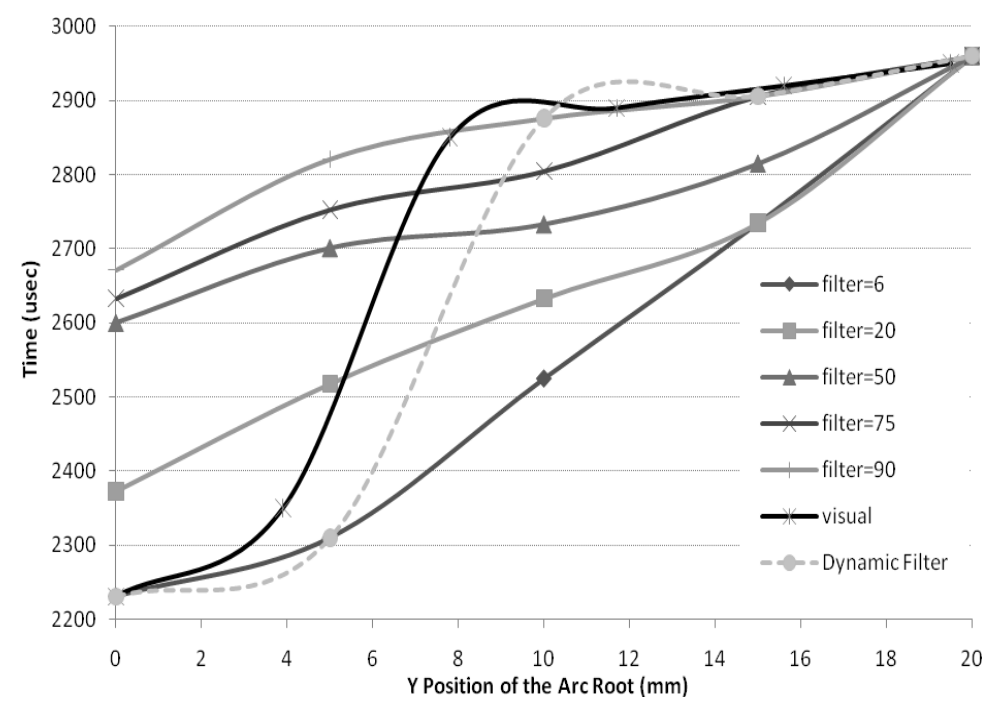

Fig 8. Parametric study of arc root position with filter values. 


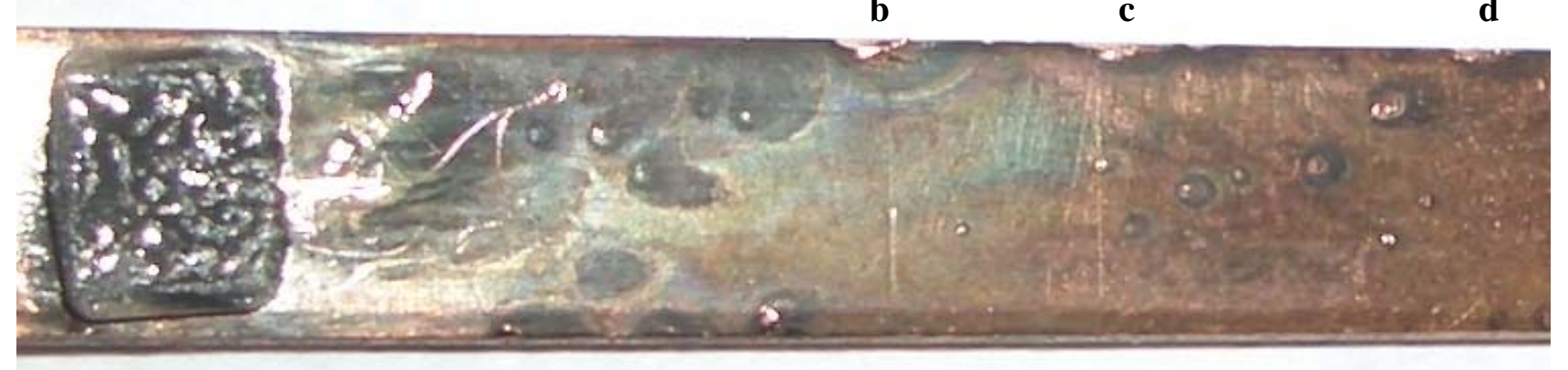

$\mathbf{a}$

Fig.9 Showing the cathode arc runner after the test shown in Fig.5, with highlighted regions where the surface has melted.

\section{Arc Root Motion}

With reference to (2); the evaluation of the arc root position (y) is influenced by low light levels on fiber positions at a distance from the zero position, for example at fiber positions AS and AF in Fig.2. To overcome this limitation a filter can be applied to the calculation such that light levels below the filter value are set to zero. The influence of the filter value on the cathode root position is shown in the Fig. 7 and 8. Fig 7 shows the cathode root position determined using (2), for two filter values, 0 and 90 . For the filter value 0 , at $2 \mathrm{~ms}$ that cathode is shown to be $5 \mathrm{~mm}$ from the contact region, with an erratic position. This does not correspond to the visual images where the arc is shown to be present in the region of the contacts until approximately $2.8 \mathrm{~ms}$. By increasing the filter value to 90 , the cathode root passes the $5 \mathrm{~mm}$ displacement line at 2.85ms. The position of the arc root with filter value 90 is also shown in Fig 5, for direct comparison with the arc voltage; this shows that although the arc voltage increases uniformly the cathode root position is characterized by periods when the arc root is stationary. Fig. 8 shows the results of a parametric study of the filter value and compares the predicted arc root positions with the visual record. It shows that as the filter value increases, the time at which the arc root passes a given position $<20 \mathrm{~mm}$ increases. Irrespective of the filter value, the displacements converge at 20mm, indicating that the filter value is important in determining the lower displacement values, this is also shown in Fig.7. The visual record is approximated from the movie sequence; it shows the cathode arc root moving rapidly after $2.3 \mathrm{~ms}$ to a position approximately $10 \mathrm{~mm}$ from the contact region, the arc then appears to move relatively slowly to the $20 \mathrm{~mm}$. The filter value which best matches the visual record when the displacement is $>10 \mathrm{~mm}$ is 90 . This filter level excludes the data at the start of the arcing process when the light levels are low, thus leading to an error in the estimation over the first $7 \mathrm{~mm}$ of the arc 
motion. To overcome this limitation the results of a dynamic filter level are shown in Fig.8. This shows a similar displacement characteristic to the visual record. The combination of the intensity plotting method identified in (2), with the 8 bit light intensity resolution results in resolution in the arc root plotting method of 10nm, when the optical fibers are mounted 3.9mm apart. To enable a correlation with arc root plotting, Fig 9 shows an image of the cathode runner after the experiment. It shows the Ag/C contact on the left and a number of regions where there is clear melting of the surface. These 4 positions a,b.c,d are plotted as wear areas in Fig.7. They show a correlation with the periods when the cathode arc root is stationary.

\subsection{Experiment 2.}

The network parameters have been adjusted in order to feed the left pole of the breaker in Fig.4 with $400 \mathrm{~V}$, 5.8kA prospective current, and 0.54 power factor. These values have been chosen as they do not represent a severe testing condition for the breaker. Eight frames are shown in Fig. 10 while in Fig. 11 shows the corresponding arc voltage and current, referenced with the corresponding frame numbers. Arc visualization is based on method 3, using (1). The arc is ignited in frame 1, with the step in arc voltage. The arc elongates from frame 2 to frame 6 and moves into the arc chamber. Frame 6 shows only a small fraction of the arc since the current is decreasing rapidly, and only the hottest part of the plasma is visible. In this experiment, the mobile contact played the role of the cathode, which is known to be considerably hotter than the anode. This can be appreciated in the last frames, relevant to a time period when the arc is cooler and thus the differences in light emission in the two arc roots are not covered by saturation. In frame 7 a sudden drop in the arc voltage is observed. The AIS clearly shows that this happens in correspondence with a back re-ignition of the arc in the central region of the chamber. This type of phenomena is problematic if it occurs just before the current zero, since it can cause thermal reignition, which may lead to another semi-cycle of arcing. A physical explanation of the phenomenon is related to the chaotic fluctuations of the arc plasma in the chamber, and the position of the plasma in splitter plates. When most of the arc plasma is in the splitter plates, that is, when it occupies the upper most third of the frames, then a number of cathodic and anodic arc root spots are activated, each providing an additional resistance (and voltage drop) to the arc. If the arc plasma cloud is mostly outside the splitter plates (shown in frame 7), its resistance is only due to just one cathodic and one anodic arc root spot on the runner and contact regions. Finally, the extinction of the arc is observed in frame 8. 

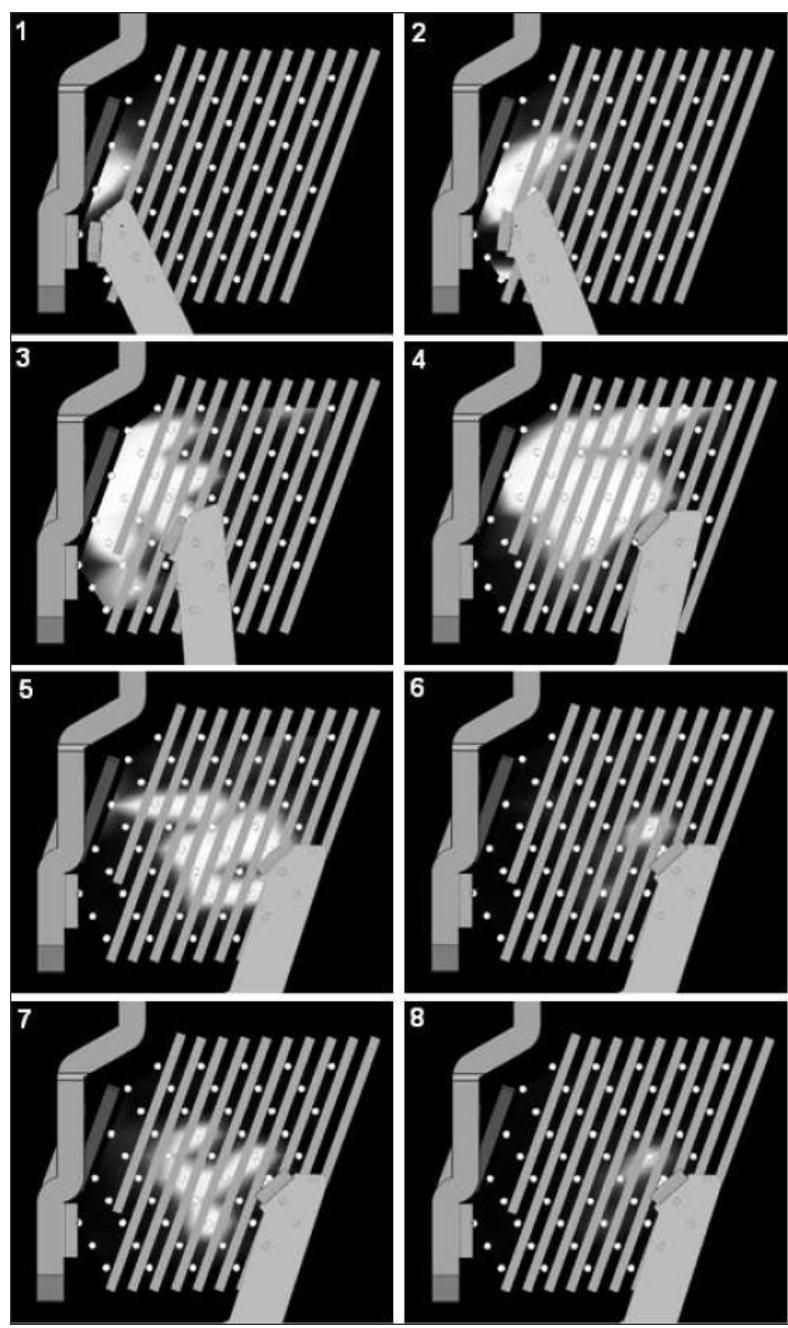

Fig. 11, Arc evolution during a short circuit as observed with fiber optics (test I). 


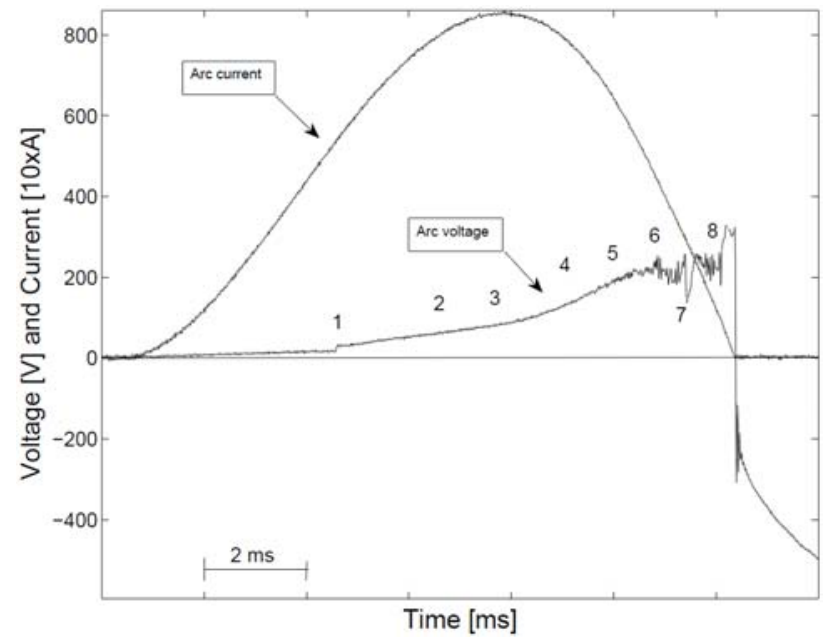

Fig. 12 Arc voltage and arc current vs. time (test I).

\section{Conclusions}

A portable arc imaging system has been developed based on an array of optical fiber forming an optical head, which can be mounted to investigate the high speed plasma motion in arc chambers used for circuit breaker technology. The system has been shown to create images of the arc motion at framing frequencies of $1 \mathrm{MHz}$, with 8 bit light intensity resolution. The improved bit resolution over other methods has allowed for two new methods of imaging the arc motion, the first based on the fiber position while the second is based on linear interpolation. The method for detecting the arc root motion has been reviewed and been shown to be in error in previous studies. The method has been advanced here by using filter cut off values which minimize the errors causes at low light levels. The new methods have been compared with a visual record of the arc motion, which shows the cathode arc root stationary for periods. This has also been correlated with a study of the wear region of the cathode runner. The system has been applied to the optimization process for a commercial circuit breaker device. This has shown the ability of the system to detect arc re-ignition phenomena after the current zero.

\section{References}

[1] Hong, D., Sandolache, G., Bauchire, J., Gentils, F, and Fleurier, C. “A New Optical Technique for Investigations of Low-Voltage Circuit Breakers”, IEEE Trans. on Plasma Science, 33, 2, 2005, pp. 976-981.

[2] C Brdys, J P Toumazet, A Laurent and J L Ponthenier, “Optical and magnetic diagnostics of the electric arc dynamics in a low voltage circuit breaker”, Institute of Physics, Measurement Science and Technology, Vol 13, pp1146-1153, 2002. 
[3] Shea J. “Gassing arc chamber wall material effect on post current-zero recovery voltage breakdown”, IEEE Trans. Components and Packaging Technologies, Vol 27, pp42-50, 2004.

[4] P.M. Weaver, J.W. McBride, and C.C. Siew, “Integrated measurement system for high speed unsteady plasma flow and its application to electric arcs”, IEE Proceedings - Science, Measurement and Technology, Vol.150, No. 4, pp. 153-160, 2003.

[5] J.W. McBride and P.M. Weaver, "Review of arcing phenomena in low voltage current limiting circuit breakers”, IEE Proceedings - Science, Measurement and Technology, Vol. 148, No. 1, pp. 1-7, 2001.

[6] P.M.Weaver, K. Pechrach and J.W. McBride, “Arc root mobility on piezoelectrically actuated contacts in miniature circuit breakers”, IEEE Transactions on Components and Packaging Technologies, Vol. 28, No. 4, pp. 734-740, 2005. [7] P.M. Weaver, K. Pechrach and J.W. McBride, “The energetics of gas flow and contact erosion during short circuit arcing”, IEEE Transactions on Components and Packaging Technologies, Vol. 27, No. 1, pp. 51-56, 2004.

[8] J.W. McBride, K. Pechrach and P.M. Weaver, “Arc motion and gas flow in current limiting circuit breakers operating with a low contact switching velocity”, IEEE Transactions on Components and Packaging Technologies, Vol. 25, No. 3, pp. 427-433, 2002.

[9] J.W. McBride, K. Pechrach, and P.M. Weaver, “Arc root commutation from moving contacts in low voltage devices,” IEEE Transactions on Components and Packaging Technologies, Vol. 24, No. 3, pp. 331-336, 2001.

[10] www.taicaanresearch.com

[11] B. Delaunay, "Sur la sphere vide",1934, VOLUME 7, Izvestia Akademii Nauk SSSR, Otdelenie Matematicheskikh i Estestvennykh Nauk", pp793—800

[12] www.taicaan.com 